Eletrodos Impressos Construídos por Serigrafia Utilizando Negro de Fumo como Material Condutor

\author{
dos Santos, S. M. V.; Oliveira, P. R.; Oliveira, M. C.; Bergamini, M. F.;* \\ Marcolino-Jr, L. H.
}

Rev. Virtual Quim., 2017, 9 (2), 626-640. Data de publicação na Web: 7 de abril de 2017

http://rvq.sbq.org.br

\title{
Screen-Printed Electrodes Constructed Using Carbon Black as Conductive Material
}

Abstract: The use of screen-printed electrodes has become attractive in the last years, mostly in function of its versatility and low-cost. In the present work, were developed screen-printed electrodes using a simple production methodology (silk-screen) and alternative materials (carbon black and acetate of cellulose). Screen printed electrodes prepared with $75 \%(\mathrm{~m} / \mathrm{m})$ of carbon black and $25 \%(\mathrm{~m} / \mathrm{m})$ of cellulose acetate had shown the best performance and they were employed for determination of ascorbic acid by chronoamperometry and cadmium and lead ions using anodic stripping voltammetry. Limit of detection of $3.1 \times 10^{-5} \mathrm{~mol} \mathrm{~L}^{-1}$ and relative deviation of $1.9 \%$ were found for determination of ascorbic acid and linear dynamic range from $8.0 \times 10^{-7}$ to $3.2 \times 10^{-5} \mathrm{~mol} \mathrm{~L}^{-1}$ and $1.0 \times 10^{-6}$ to $6.3 \times 10^{-5} \mathrm{~mol} \mathrm{~L}^{-1}$ were verified for cadmium and lead ions respectively.

Keywords: Screen printed electrodes; carbon black; voltammetric sensors.

\section{Resumo}

O uso de eletrodos impressos tem se tornado bastante atrativo nos últimos anos em função principalmente de sua versatilidade e baixo custo. No presente trabalho, foram desenvolvidos eletrodos impressos utilizando metodologias de produção simples (serigrafia) e materiais alternativos (negro de fumo e acetato de celulose). Os eletrodos impressos preparados com $75 \%(\mathrm{~m} / \mathrm{m})$ de negro de fumo e $25 \%(\mathrm{~m} / \mathrm{m})$ de acetato de celulose apresentaram o melhor desempenho e foram empregados na determinação cronoamperométrica de ácido ascórbico e de íons chumbo e cádmio utilizando a voltametria de redissolução anódica. 0 limite de detecção de $3,1 \times 10^{-5} \mathrm{~mol} \mathrm{~L}^{-1}$ e os desvios relativos inferiores a $1,9 \%$ foi verificado para a determinação de ácido ascórbico e faixas lineares de resposta de $8,0 \times 10^{-7}$ a 3,2 $\times 10^{-5} \mathrm{~mol} \mathrm{~L}^{-1} \mathrm{e}$ $1,0 \times 10^{-6}$ a $6,3 \times 10^{-5} \mathrm{~mol} \mathrm{~L}^{-1}$ foram encontradas para íons cádmio e chumbo, respectivamente.

Palavras-chave: Eletrodos impressos; negro de fumo; sensores voltamétricos.

* Universidade Federal do Paraná, Laboratório de Sensores Eletroquímicos (LabSensE), Departamento de Química, CEP 81531-980, Curitiba-PR, Brasil.

Mbergamini@ufpr.br

DOI: $10.21577 / 1984-6835.20170037$

Rev. Virtual Quim. |Vol9| |No.2 | |626-640| 


\section{Eletrodos Impressos Construídos por Serigrafia Utilizando Negro de Fumo como Material Condutor}

\section{Sergio M. V. dos Santos, Paulo R. de Oliveira, Michelle C. de Oliveira, Márcio F. Bergamini, * Luiz H. Marcolino-Jr}

Universidade Federal do Paraná, Laboratório de Sensores Eletroquímicos (LabSensE),

Departamento de Química, CEP 81531-980, Curitiba-PR, Brasil.

* bergamini@ufpr.br

Recebido em 1 de junho de 2016. Aceito para publicação em 7 de abril de 2017

\section{Introdução}

\section{Parte Experimental}

2.1. Reagentes

2.2. Preparação da tinta condutora

2.3. Construção dos Eletrodos impressos

2.4. Caracterização morfológica e eletroquímica dos El's

2.5. Avaliação da potencialidade analítica dos El's na determinação de íons metálicos ( $\mathrm{Cd}(\mathrm{II})$ e $\mathrm{Pb}(\mathrm{II}))$ e ácido ascórbico

\section{Resultados e Discussão}

3.1. Caracterização morfológica e voltamétrica dos eletrodos impressos

3.2. Determinação voltamétrica de íons $\mathrm{Cd}(\mathrm{II})$ e $\mathrm{Pb}(\mathrm{II})$ por voltametria de redissolução anódica

3.3. Determinação cronoamperométrica de ácido ascórbico em formulações farmacêuticas

\section{Conclusão}

\section{Introdução}

Os eletrodos impressos (EI) têm sido amplamente empregados como uma alternativa ao uso de eletrodos convencionais em eletroanálises. A substituição apresenta a vantagem de oferecer sistemas mais simples, menores, com arranjo de eletrodos mais compactos e construídos sobre um único suporte, o que é adequado para o desenvolvimento de sensores portáteis. Outras vantagens no uso deste tipo de dispositivo estão associadas ao fato de serem produzidos em batelada, barateando o custo de produção e permitindo que sejam utilizados como descartáveis, o que diminui as etapas de limpeza entre as análises e evita problemas de envenenamento da superfície do eletrodo. ${ }^{1,2}$

Diversas técnicas são utilizadas para a 
construção de eletrodos impressos, tais como a impressão por microcontato,,$-\frac{3}{}$ a litografia,, a impressão por jato de tinta, ${ }^{5}$ e a serigrafia. ${ }^{6}$ Além da geometria/configuração e da composição do material usado na confeç̧ão dos eletrodos impressos, a escolha da técnica de preparação permite a construção de dispositivos com características particulares que podem levar a diferentes aplicações. ${ }^{7-10}$ Dentre as técnicas mais utilizadas para a impressão de Els, a serigrafia ou "silk-screen" é uma opção simples e versátil que vem sendo explorada com sucesso desde a década de $1990 .^{1,2,11}$ Esta metodologia pode ser aplicada facilmente em qualquer laboratório, uma vez que não exige equipamentos sofisticados e/ou de grande porte para o processo de impressão.

Os componentes básicos de um El são um suporte não condutor (vidro, plástico ou cerâmico), uma camada condutora impressa (material condutor/ aglutinante) sobre o suporte inerte. A impressão da camada condutora é realizada passando a tinta condutora através de um molde, que consiste em uma tela com a configuração do arranjo de eletrodo definida. Na maioria das vezes, esta camada é parcialmente coberta por uma segunda camada isolante para definir uma área de contato elétrico numa extremidade e a superfície do eletrodo.

Dentre as diversas frentes de investigações sobre os Els, um dos principais desafios está relacionado à de obtenção da tinta condutora que apresente condutividade elétrica e viscosidade adequada ao processo de serigrafia. Algumas das tintas condutoras que são disponíveis comercialmente foram originalmente desenvolvidas para a confecção de eletrodos, sensores, circuitos impressos entre outros dispositivos, o preço e as dificuldades no processo de importação dessas tintas dificultam sua ampla utilização. Nesse contexto, o preparo de tintas caseiras ou Homemade Inks (HMIs) surge como uma alternativa interessante, ampliando a versatilidade na confecção dos Els e também na diminuição nos custos de produção. Com relação ao componente condutor, o carbono, em suas formas condutoras, tem sido amplamente empregado como matériaprima para a preparação de tintas. ${ }^{12}$ Dentro deste contexto, uma excelente alternativa, e ainda pouco explorada é a utilização de negro de fumo pois, ele possui um custo significativamente baixo e uma ampla disponibilidade em comparação a outros materiais comumente utilizados. ${ }^{13}$

O Negro de fumo (NF) ou Carbon Black (CB) é um termo genérico usado para identificar uma ampla variedade de materiais carbonáceos em forma de partículas esféricas com tamanhos na faixa de 10-100 nm, produzidas através da decomposição térmica controlada de hidrocarbonetos aromáticos. ${ }^{14}$ Existem muitos tipos de NF comerciais, eles se diferem pelo tipo de tecnologia empregada na obtenção e pela qualidade do produto final. Estes materiais possuem concentrações acima de $90 \%$ de carbono elementar e propriedades físicas bem estabelecidas, de acordo com seu processo de fabricação. ${ }^{15} \mathrm{~A}$ aplicação deste material na área industrial é extremamente ampla podendo variar desde o seu uso como material adsorvente, pigmentos industriais ou até mesmo utilizado na construção de células a combustível, baterias a base de lítio e sódio ${ }^{16-18}$ e mais recentemente, como agente modificador em eletrodos. ${ }^{10,19,20}$ São escassas informações sobre a potencialidade do NF como material condutor principal na preparação de tintas condutoras visando a construção de El.

Este trabalho tem como objetivo construir eletrodos impressos de baixo custo empregando a serigrafia e utilizando negro de fumo (NF) como material condutor, investigar as características eletroquímicas dos dispositivos construídos e avaliar a potencialidade dos eletrodos produzidos como sensores eletroquímicos na determinação de compostos orgânicos e inorgânicos.

\section{Parte Experimental}

\subsection{Reagentes}


Os reagentes e as soluções com padrão analítico (P.A.) foram utilizados sem previa purificação, sendo eles: Cloreto de potássio $(\mathrm{KCl})$, hidróxido de sódio $(\mathrm{NaOH})$, iodeto de potássio (KI), iodo $\left(\mathrm{I}_{2}\right)$ e tiossulfato de sódio $\left(\mathrm{Na}_{2} \mathrm{~S}_{2} \mathrm{O}_{3}\right)$ foram adquiridos da Vetec. $\mathrm{O}$ ácido ascórbico $\left(\mathrm{C}_{6} \mathrm{H}_{8} \mathrm{O}_{6}\right)$, o nitrato de chumbo $\left(\mathrm{Pb}\left(\mathrm{NO}_{3}\right)_{2}\right)$, o dicromato de potássio $\left(\mathrm{K}_{2} \mathrm{Cr}_{2} \mathrm{O}_{7}\right)$ e o amido solúvel $\left(\mathrm{C}_{12} \mathrm{H}_{22} \mathrm{O}_{11}\right)$ foram adquiridos da Sigma-Aldrich; o ácido sulfúrico $\left(\mathrm{H}_{2} \mathrm{SO}_{4}\right)$ e o ácido clorídrico da FMaia-Gold, o hexacianoferrato (III) de potássio $\left(\mathrm{K}_{3}\left[\mathrm{Fe}(\mathrm{CN})_{6}\right]\right)$ da Ecibra, o ácido acético glacial $\left(\mathrm{CH}_{3} \mathrm{COOH}\right)$ da Isofar, o acetato de sódio $\left(\mathrm{CH}_{3} \mathrm{COONa}\right)$ da J.T. Baker e o nitrato de potássio $\left(\mathrm{KNO}_{3}\right)$ da Synth. A água destilada e deionizada foi utilizada na preparação das soluções aquosas. As soluções estoque foram preparadas por pesagem dos sólidos correspondentes, utilizando uma balança analítica moledo Modelo AL204 com precisão de $\pm 0,1 \mathrm{mg}$ e posteriormente diluídas em água ultra-pura (Milli-Q). As soluções com menores concentrações $\left(\mathrm{Cd}^{2+} 4,0 \times 10^{-7}\right.$ a 9,0 $\times 10^{-5} \mathrm{~mol} \mathrm{~L}^{-1} ; \mathrm{Pb}^{2+} 8,0 \times 10^{-7}$ a $1,6 \times 10^{-4} \mathrm{~mol} \mathrm{~L}^{-1}$ e AA $1,0 \times 10^{-6}$ a $5,7 \times 10^{-4} \mathrm{~mol} \mathrm{~L}^{-1}$ ) foram preparadas por diluição da solução estoque utilizando micropipetas de volumes reguláveis.

\subsection{Preparação da tinta condutora}

As tintas condutoras foram preparadas utilizando Negro de Fumo Monarch $570^{\circledR}$ com diâmetro médio de 24 nanômetros, acetato de celulose Sigma-Aldrich, acetona e ciclohexanona Vetec, de grau analítico. Os solventes foram escolhidos de acordo com trabalhos previamente relatados na literatura ${ }^{10-12}$ sendo utilizada uma mistura de acetona e cicloexanona avaliadas em diferentes proporções da mistura acetona/ciclohexanona: $0 / 100,20 / 80,40 / 60$, $60 / 40,80 / 20$ e $100 / 0(\% \mathrm{~m} / \mathrm{m})$, para a solubilização do material aglutinante (acetato de celulose) da tinta. Para avaliar qual a melhor composição da tinta condutora foi realizado um estudo variando a porcentagem em massa de acetato de celulose (100 - $\mathrm{x} \%$ ) e NF (x \%). As quantidades de NF estudadas foram de $70,75,80,85$ e $90 \%(\mathrm{~m} / \mathrm{m})$. Cada conjunto de eletrodos confeccionado foi submetido a varreduras de potenciais (voltametria cíclica) com velocidade de varredura de $25 \mathrm{mV} \mathrm{s}^{-1}$ na presença de 5,0 x $10^{-3} \mathrm{~mol} \mathrm{~L}^{-1}$ de ferricianeto de potássio $\left(\mathrm{K}_{3}\left[\mathrm{Fe}(\mathrm{CN})_{6}\right]\right)$ e $0,1 \mathrm{~mol} \mathrm{~L}^{-1}$ de nitrato de potássio $\left(\mathrm{KNO}_{3}\right)$. A escolha da melhor composição do solvente, bem como da melhor proporção entre NF e acetato de celulose foi avaliada com base na definição dos processos faradaicos, referentes à oxidação e redução do par ferrocianeto/ferricianeto, e a relação entre a diferença de potencial de pico anódico e catódico (reversibilidade).

\subsection{Construção dos Eletrodos Impressos} (EI)

Os eletrodos foram impressos em placas de PVC de $120 \mathrm{~cm}$ por $60 \mathrm{~cm}$ com $0,7 \mathrm{~mm}$ de espessura, com o auxílio de uma tela de serigrafia de nylon ${ }^{\circledR}$ contendo 120 fios por $\mathrm{cm}^{2}$ e rodo de serigrafia, todos adquiridos em lojas especializadas da cidade de Curitiba. As etapas envolvidas na preparação dos eletrodos estão ilustradas na Figura 1. As placas de PVC (Fig. 1-A) foram previamente limpas com acetona e a impressão foi realizada utilizando a tela de nylon ${ }^{\circledR}$ (Fig. 1B) e um rodo, usado para arrastar e pressionar a tinta através do molde impresso na tela. Na primeira etapa, realizou-se a impressão da tinta condutora por um molde apropriado sobre a placa de PVC (Fig. 1-C). Após a impressão é necessário aguardar alguns minutos para que a tinta condutora seque em temperatura ambiente. $O$ número de camadas da tinta condutora foi avaliado de acordo com o perfil voltamétrico obtido para os eletrodos com diferentes números de camadas em uma solução de hexacianoferrato (III) de potássio. A partir das medidas de voltametria cíclica foram considerados: a definição dos picos de 
corrente e a diferença de potencial entre os picos anódico e catódico. A seguir, realizou-se a etapa de delimitação da área geométrica dos eletrodos, o procedimento foi o mesmo utilizado anteriormente, porém, utilizando-se uma segunda tela de impressão contendo pequenos círculos com área de aproximadamente $2,0 \mathrm{~mm}$ de diâmetro em uma das extremidades (área dos eletrodos) e livre na outra extremidade para o contato elétrico com o equipamento de medida (Fig 1-D). A tinta escolhida para a camada isolante dos Els foi uma tinta vinilíca do fabricante i9 para impressão serigráfica, e uma segunda impressão utilizando-se esmalte sintético Coralit $^{\circledR}$ da fabricante Coral (Fig 1-E). O esmalte sintético impresso passou por um processo de cura, sob fluxo de ar, a uma temperatura de aproximadamente $40^{\circ} \mathrm{C}$, até que estivesse completamente seco. Este procedimento é realizado para garantir que não existam falhas na estrutura da camada isolante, que possam permitir a permeação da solução externa ao eletrodo. O eletrodo final está apresentado na Fig 1-E.

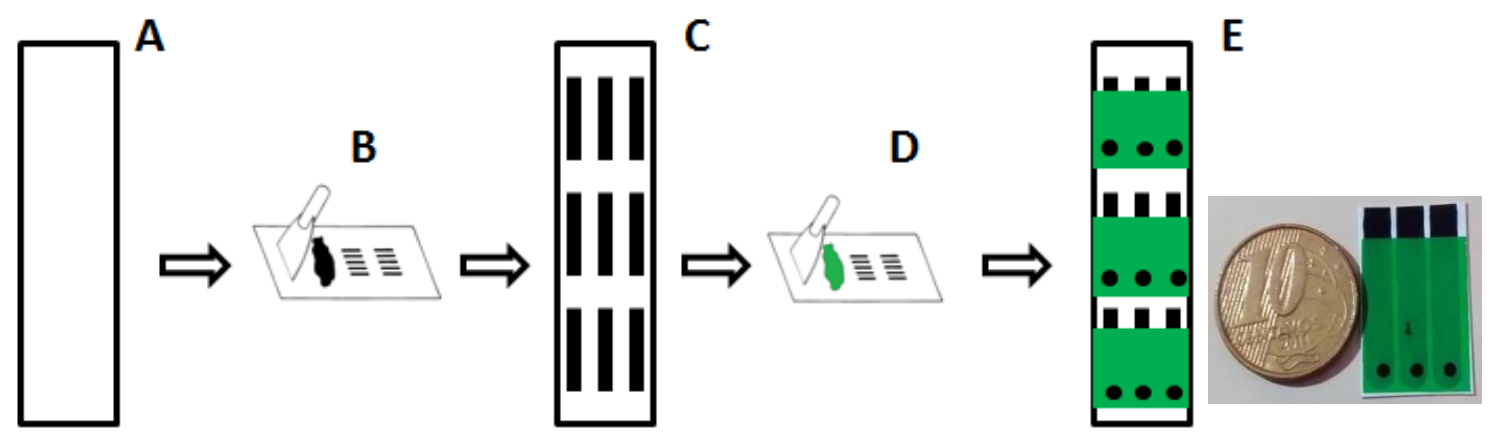

Figura 1. (A) Placa de PVC previamente limpa; (B) Impressão da tinta condutora; (C) El com a tinta condutora; (D) impressão da tinta isolante e (E) El pronto. (adaptado da referência 12)

\subsection{Caracterização morfológica e eletroquímica dos El's}

A análise morfológica dos El's foi realizada por microscopia eletrônica de varredura (MEV). As análises foram realizadas utilizando o Microscópio eletrônico de varredura marca JEOL, modelo JSM-6360LV. As análises foram realizadas no Centro de Microscopia Eletrônica da UFPR.

As medidas eletroquímicas (voltametria cíclica, voltametria linear e cronoamperometria) foram realizadas por um potenciostato/galvanostato $\mu$ Autolab ${ }^{\circledR}$ Type III, a coleta de dados foi obtida com auxílio do software NOVA 7.0 EcoChemie ${ }^{\circledR}$. Os dados coletados foram tratados com o software Origin $\mathrm{PRO}^{\circledR}$. As medidas foram realizadas em uma célula eletroquímica de $10,0 \mathrm{~mL}$ utilizando o eletrodo impresso proposto. A fim de comparar os resultados obtidos com o dispositivo proposto, algumas medidas foram realizadas utilizando um conjunto de três eletrodos, sendo, eletrodo de trabalho, o eletrodo impresso a base de carbono, o eletrodo de referência de $\mathrm{Ag} / \mathrm{AgCl} / \mathrm{KCl} 3,0$ mol $L^{-1}$ e um eletrodo de platina que foi utilizado como eletrodo auxiliar.

Os El's foram avaliados em solução de cloreto de potássio $0,1 \mathrm{~mol} \mathrm{~L}^{-1}(\mathrm{KCl})$ como eletrólito suporte contendo hexacianoferrato (III) de potássio na concentração de 5,0 × 10 $0^{-3}$ mol L $\mathrm{L}^{-1}$. O comportamento eletroquímico dos El's foi avaliado com base nos valores de corrente e de potencial de pico observados para os processos de oxidação/redução dos ânions ferrocianeto/ferricianeto contra um eletrodo referência convencional de $\mathrm{Ag} / \mathrm{AgCl} / \mathrm{KCl} 3,0 \mathrm{~mol} \mathrm{~L}^{-1}$ e eletrodo de platina como eletrodo auxiliar. Parâmetros como repetibilidade (nove medidas com o mesmo dispositivo) e reprodutibilidade (nove dispositivos diferentes) foram avaliados com base na definição dos processos faradaicos 
referentes à oxidação e redução do par ferrocianeto/ferricianeto, e na diferença entre os potenciais de pico anódico e catódico (reversibilidade).

2.5. Avaliação da potencialidade analítica dos El's na determinação de íons metálicos ( $\mathrm{Cd}(\mathrm{II})$ e $\mathrm{Pb}(\mathrm{II}))$ e ácido ascórbico

A avaliação do desempenho analítico dos Els fabricados foi investigado na préconcentração e determinação de íons $\mathrm{Pb}$ (II) e $\mathrm{Cd}$ (II) empregando voltametria de redissolução anódica. Para as medidas foi empregada uma velocidade de varredura de $20 \mathrm{mV} \mathrm{s}^{-1}$, tempo de pré-concentração de 120 segundos e varredura de potencial de $-1,3 \mathrm{~V}$ até $-0,6 \vee$ (vs. $C_{\text {impresso }}$ ). Todas as medidas foram realizadas utilizando os três eletrodos impressos de carbono. Para a avaliação do melhor $\mathrm{pH}$ de medida foram realizadas medidas em acetato de sódio $0,1 \mathrm{~mol} \mathrm{~L}^{-1} \mathrm{com}$ $\mathrm{pH}$ ajustado no intervalo entre 5,0, e 7,0. Curvas analíticas foram construídas para cada um dos analitos a fim de se obter os parâmetros de mérito analítico: faixa de resposta linear (LDR - Linear Dynamic Range), limite de detecção ( $L O D$ - Limit of detection), limite de quantificação ( $L O Q$ Limit of Quantification) e sensibilidade de calibração. A linearidade ou faixa de resposta linear de trabalho foi obtida por padronização externa e considerada com base na relação linear entre o sinal e a concentração, os valores de limite de deteç̧ão e quantificação foram calculados como LOD $=3 * \mathrm{SD}_{\text {branco }} / \mathrm{b}$ e $\mathrm{LOQ}=$ $10 * \mathrm{SD}_{\text {branco }} / \mathrm{b}$ onde: $\mathrm{SD}_{\text {branco }}$ é o desvio padrão de 10 medidas consecutivas do branco e b é - coeficiente angular (sensibilidade de calibração) da curva analítica ${ }^{21}$. 0 procedimento foi empregado na determinação de íons metálicos em amostras de água de torneira enriquecidas com íons $\mathrm{Cd}(\mathrm{II})$ e $\mathrm{Pb}$ (II) em concentrações próximas ao limite permitido pela resolução CONAMA 430/2011 sobre lançamento de efluentes, sendo $0,5 \mathrm{mg} \mathrm{L}^{-1}$ para $\mathrm{Pb}(\mathrm{II})$ e $0,2 \mathrm{mg} \mathrm{L}^{-1}$ para cádmio $\mathrm{Cd}(\mathrm{II})$.

Os El's construídos também foram empregados na determinação amperométrica de ácido ascórbico (AA). A influência do $\mathrm{pH}$ da solução de medida sobre a resposta voltamétrica foi realizada por voltametria cíclica em meio de acetato de sódio $0,1 \mathrm{~mol} \mathrm{~L}^{-1} \mathrm{com} \mathrm{pH}$ ajustado no intervalo de 2,0 a 6,0 na presença de 5,0 $\mathrm{x}$ $10^{-4} \mathrm{~mol} \mathrm{~L}^{-1}$ de AA. Parâmetros de mérito analítico foram obtidos a partir de curvas analíticas construídas por cronoamperometria (a influência do potencial aplicado na medida amperométrica foi investigada variando-se o potencial aplicado em um intervalo de 0,25 a 0,65 V (vs. $\left.C_{\text {impresso) }}\right)$. Neste estudo, foi utilizado o conjunto impresso de eletrodo de trabalho, auxiliar e referência de carbono. O mesmo conjunto foi utilizado para a determinação de ácido ascórbico em amostras comerciais de vitamina C efervescente.

\section{Resultados e Discussão}

\subsection{Caracterização morfológica e voltamétrica dos eletrodos impressos}

A construção de um eletrodo impresso, em sua maioria, consiste na imobilização de uma tinta condutora em um substrato inerte. Neste trabalho a tinta condutora consiste em um compósito a base de negro de fumo aglutinado homogeneamente em acetato de celulose. A escolha do melhor solvente ou mistura de solventes empregados para dissolver o acetato de celulose é de extrema importância, uma vez que este irá conferir o aspecto da tinta a ser impressa.

A escolha do melhor solvente foi feita pela avaliação de diferentes parâmetros, como por exemplo, a solubilização do acetato de celulose, adequação ao processo silk-screen e homogeneidade da tinta. Foi observado que - aglutinante é solubilizado com mais facilidade em uma mistura de solventes com 
uma maior proporção de acetona $\mathrm{e}$, respectivamente, $\mathrm{o}$ decréscimo da quantidade de ciclohexanona. Entretanto, o aumento da proporção de acetona leva a uma volatilização mais rápida do solvente, diminuindo o tempo de manuseio da tinta, gerando também menor homogeneidade e aparecimento de falhas na tinta depositada sobre o suporte de PVC após a secagem. Para maiores quantidades de ciclohexanona a solubilidade da tinta é menos eficaz o que inviabiliza o emprego deste solvente.

Utilizando $20 \%$ em volume de acetona e $80 \%$ de cicloexanona ainda há dificuldade para a solubilização do acetato de celulose e, além disso, devido à baixa viscosidade da mistura (observada visualmente), é exigido um tempo maior para o preparo dos eletrodos o que torna essa composição não adequada para a produção da tinta condutora. A proporção acetona/cicloexanona de 60/40\% (v/v) permite a solubilização do aglutinante, boa volatilidade, adequação da tinta ao processo silk-screen e menor resistência elétrica. Medidas de resistência elétrica foram realizadas com auxílio de um multímetro conectado diretamente às extremidades dos eletrodos depois da secagem completa da tinta para avaliar a melhor composição de solvente. Dessa forma, foram construídos 10 eletrodos para cada uma das composições de $60 / 40 \%, 50 / 50 \%$ e $40 / 60 \%$ em volume de acetona/cicloexanona, os valores de resistência elétrica estão apresentados na Figura 2.
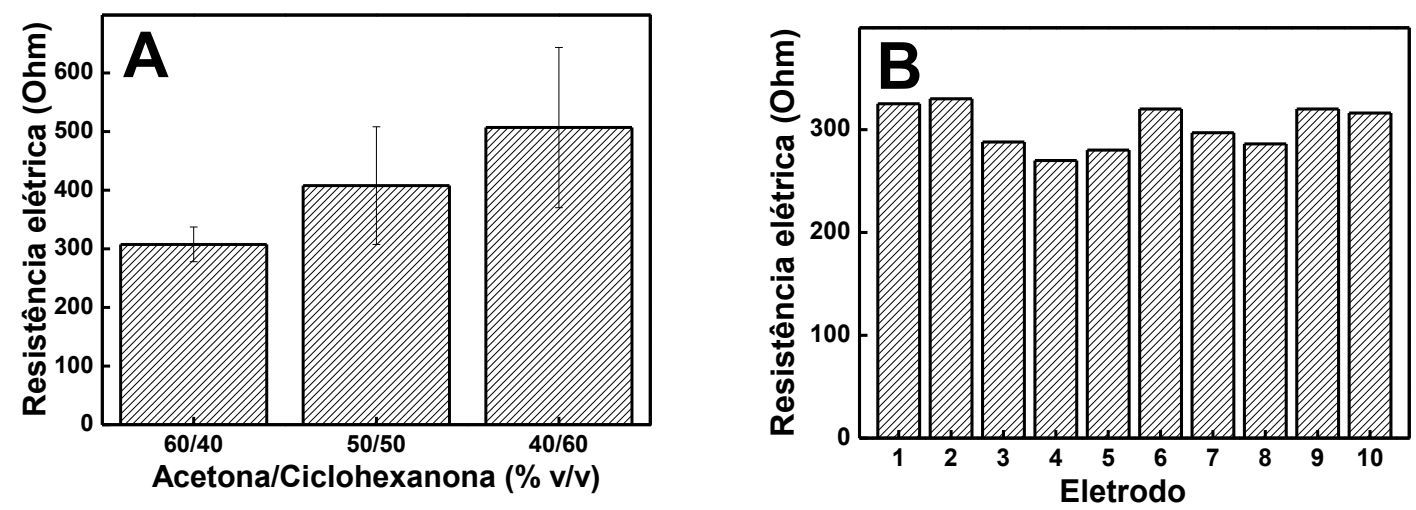

Figura 2. Valores de resistência elétrica para eletrodos preparados com diferentes proporções de solventes na tinta $(A)$ e a resistência elétrica de diferentes eletrodos preparados com a proporção 60/40\% (v/v) (B)

Com relação a melhor composição da tinta, a proporção contendo $70 \%$ NF e $30 \%$ acetato de celulose apresentou viscosidade muito elevada devido à maior quantidade de acetato de celulose quando comparada as outras composições estudadas. A maior viscosidade influenciou a passagem da tinta pela tela de impressão, inviabilizando assim a obtenção e, consequentemente, a avaliação dos Els com esta composição. Para as demais composições avaliadas, as tintas produzidas apresentaram viscosidade adequada para o processo de impressão. Os voltamogramas cíclicos obtidos para cada eletrodo confeccionado podem ser observados na Figura 3. 


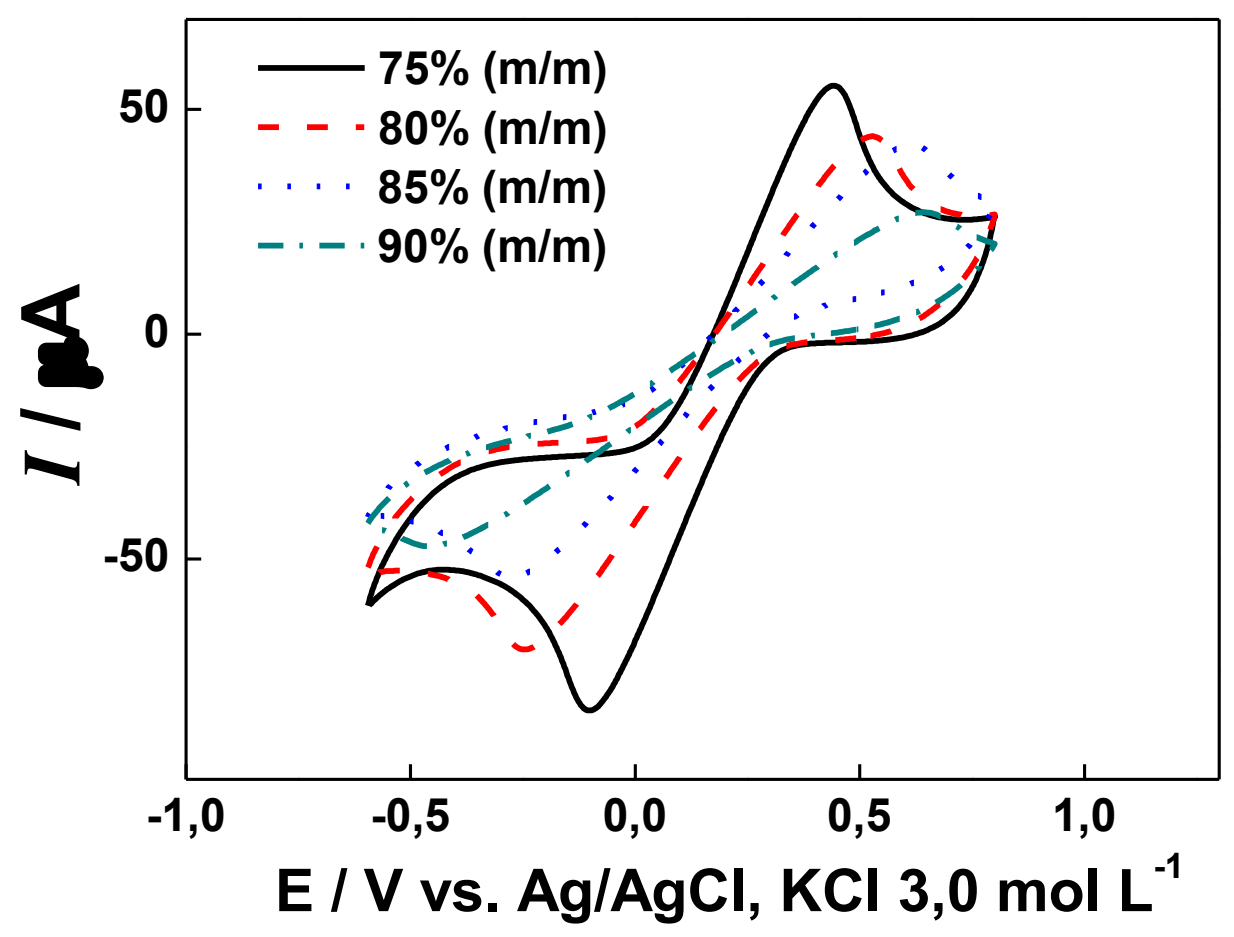

Figura 3. Voltamogramas cíclicos obtidos para íons hexacianoferrato (III) $5,0 \times 10^{-3} \mathrm{~mol} \mathrm{~L}^{-1} \mathrm{em}$ $\mathrm{KNO}_{3}$ 0,1 mol L-1 como eletrólito suporte, com velocidade de varredura de $25 \mathrm{mV} \mathrm{s}^{-1}$, para eletrodos impressos produzidos com (A) $75 \%$, (B) $80 \%$; (C) $85 \%$ e (D) $90 \%$ de negro de fumo

O perfil voltamétrico que apresentou melhor reversibilidade e melhor definição dos processos foi obtido para o eletrodo construído com as porcentagens em massa de $75 \%$ de $\mathrm{NF}$ e $25 \%$ de acetato de celulose. Em quantidades superiores de NF é observada a diminuição da reversibilidade do sistema e também a menor definição dos processos faradaicos. $O$ aumento da quantidade de NF leva ao aumento da quantidade de material condutor $\mathrm{e}$, consequentemente, seriam esperadas melhores respostas para os eletrodos assim construídos. Entretanto, maiores quantidades de NF implicam na diminuição da quantidade da fase aglutinante (acetato de celulose), que apesar de ser não condutora facilita a melhor distribuição e aderência da fase condutora no substrato. Os eletrodos construídos com elevados valores de negro de fumo apresentaram uma variação maior para medidas realizadas com diferentes dispositivos, sugerindo uma menor homogeneidade da tinta condutora.

O processo de confecção de Els por serigrafia permite a incorporação de inúmeras camadas da tinta condutora pela simples deposição, camada pós camada, influenciando significativamente no desempenho dos eletrodos. ${ }^{12}$ Assim, foram avaliados eletrodos com diferentes números de camadas de tinta condutora ( 1 a 6 ) por voltametria cíclica, como realizado para os estudos anteriores. As medidas de microscopia eletrônica de varredura (MEV) foram realizadas para investigar a morfologia da superfície dos eletrodos. A escolha do número de camadas de tinta condutora foi realizada com base no perfil voltamétrico da sonda empregada (íons ferricianeto). A Figura 4 apresenta os voltamogramas cíclicos e as imagens de MEV obtidos para os eletrodos impressos com diferentes números de camadas. 

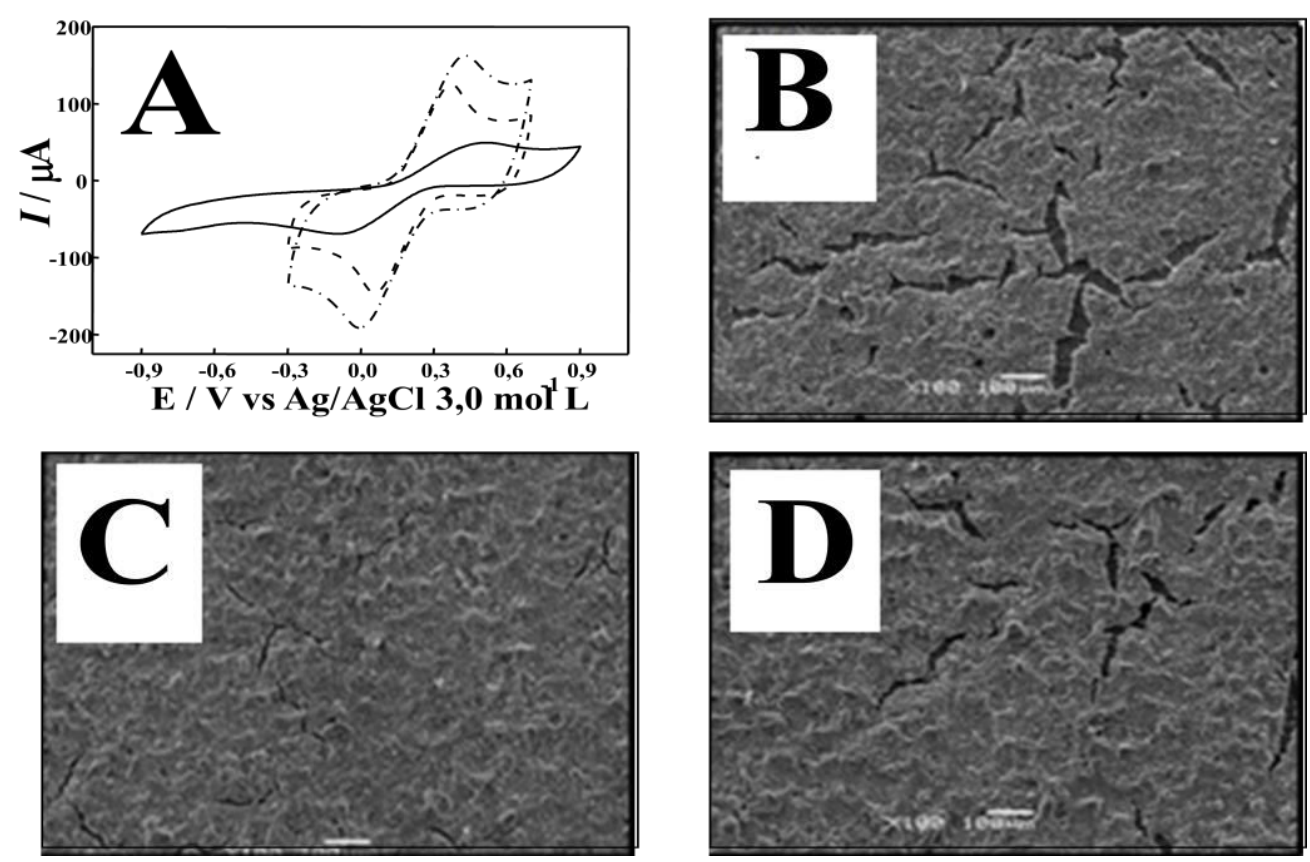

Figura 4. A) Voltamogramas cíclicos obtidos para os eletrodos impressos com 2 camadas $(-)$, 4 camadas (---) e 6 camadas (---) em solução $0,1 \mathrm{~mol} \mathrm{~L}^{-1}$ de $\mathrm{KNO}_{3}$ contendo ferricianeto de potássio $1,0 \times 10^{-3} \mathrm{~mol} \mathrm{~L}^{-1} \mathrm{e}$. Imagens de microscopia eletrônica de varredura com aumento de $100 \times$ da superfície dos eletrodos impressos em que B, C e D, correspondem aos eletrodos com 2,4 , e 6 camadas, respectivamente

Com base nos voltamogramas apresentados na Fig 4-A, a melhor definição dos processos faradaicos e uma menor separação entre os picos de corrente foram observadas para os eletrodos com maior número de camadas de tinta condutora depositadas. Esse comportamento pode estar relacionado com o melhor recobrimento da superfície do substrato de deposição (não condutor) pela tinta condutora o que promove uma maior condutividade elétrica ao eletrodo. Pelas imagens de MEV pode ser evidenciada que superfícies preparadas com menores números de camadas (Fig 4-B) apresentam "rachaduras" na superfície, o que pode promover a diminuição de condutividade. A Fig 4-C, referente ao eletrodo construído com 4 camadas, apresenta uma superfície mais homogênea e sem rachaduras em virtude do maior recobrimento do substrato. Isto promove maior condutividade ao eletrodo, como observado para os demais eletrodos com maiores números de camadas, como por exemplo, o eletrodo obtido com 6 camadas.
No entanto, esses eletrodos construídos com 6 camadas não apresentam boa repetibilidade (medidas sucessivas de um mesmo dispositivo) e/ou reprodutibilidade (diferentes dispositivos com o mesmo número de camadas). A falta de repetibilidade/reprodutibilidade entre as medidas pode estar associada ao excesso de tinta condutora depositada, evidenciada na Fig 4-D, caracterizado pelo aparecimento de pequenos grânulos com formatos similares aos dos poros da tela de impressão (retangulares). Esses grânulos, provavelmente se desprendem da superfície eletródica mudando assim a quantidade e a morfologia inicial da superfície do eletrodo. Desta forma, O El que apresentou melhor resposta voltamétrica e também melhores resultados em termos de repetibilidade/reprodutibilidade foi $O$ EI contendo 4 camadas de tinta condutora, condição adotada para estudos posteriores. 
3.2. Determinação voltamétrica de íons $\mathrm{Cd}$ (II) e $\mathrm{Pb}$ (II) por voltametria de redissolução anódica

Após avaliar as melhores condições envolvidas na construção do eletrodo impresso, foram realizados estudos para avaliar a potencialidade analítica dos dispositivos propostos na determinação de cátions metálicos empregando a voltametria de redissolução anódica. Neste estudo foram utilizadas soluções de íons $\mathrm{Cd}$ (II) $\left(1,7 \times 10^{-5}\right.$ $\left.\mathrm{mol} \mathrm{L}^{-1}\right)$ e íons $\mathrm{Pb}$ (II) $\left(1,0 \times 10^{-5} \mathrm{~mol} \mathrm{~L}^{-1}\right)$. A varredura linear foi realizada variando 0 potencial de $-1,1 \mathrm{~V}$ até $-0,6 \mathrm{~V}$, promovendo a oxidação dos íons previamente reduzidos (potencial aplicado de $-1,1 \mathrm{~V}$ ) com velocidade de varredura de $25 \mathrm{mV} \mathrm{s}^{-1}$. Os voltamogramas obtidos para este estudo são observados na Figura 5.

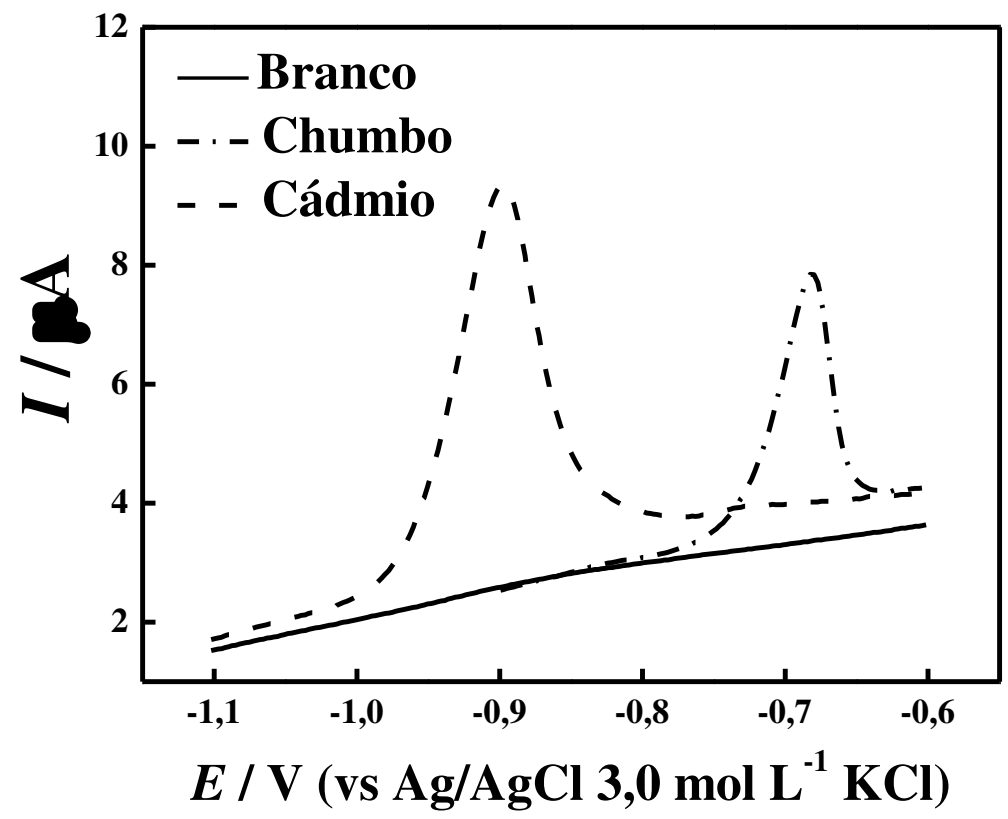

Figura 5. Voltamogramas de varredura linear obtidos para o El com a composição de $75 \%$ de NF e $25 \%$ de acetato de celulose $(\mathrm{m} / \mathrm{m})$ como tinta condutora. Tampão acetato pH 5,0 potencial de redução de $-1,1 \mathrm{~V}$ durante 60 segundos para íons $\mathrm{Cd}(\mathrm{II})\left(1,7 \times 10^{-5} \mathrm{~mol} \mathrm{~L}^{-1}\right)$ e $\mathrm{Pb}$ (II) $\left(1,0 \times 10^{-5} \mathrm{~mol} \mathrm{~L}^{-1}\right)$

Para o voltamograma obtido para o El na ausência dos íons $\mathrm{Cd}(\mathrm{II})$ e $\mathrm{Pb}(\mathrm{II})$ não foi observado nenhum processo faradaico no intervalo investigado indicando que o eletrodo não apresenta nenhum grupo eletroativo nessas condições e faixa de potencial avaliados. Após verificar a potencialidade do dispositivo proposto na determinação de íons $\mathrm{Cd}(\mathrm{II})$ e $\mathrm{Pb}(\mathrm{II})$, foram construídas curvas analíticas na presença dos respectivos íons, separadamente, em condições previamente escolhidas empregando a técnica de voltametria linear com redissolução anódica. Foram obtidos voltamogramas para diferentes concentrações de íons $\mathrm{Cd}$ (II) e $\mathrm{Pb}$ (II), de $4,0 \mathrm{x}$ $10^{-7} \mathrm{~mol} \mathrm{~L}^{-1}$ à $9,0 \times 10^{-5} \mathrm{~mol} \mathrm{~L}^{-1}$ e de $8,0 \times 10^{-7}$ mol L-1 a $1,6 \times 10^{-4} \mathrm{~mol} \mathrm{~L}^{-1}$, respectivamente. As curvas analíticas para cada íon avaliado são mostradas na Figura 6. 

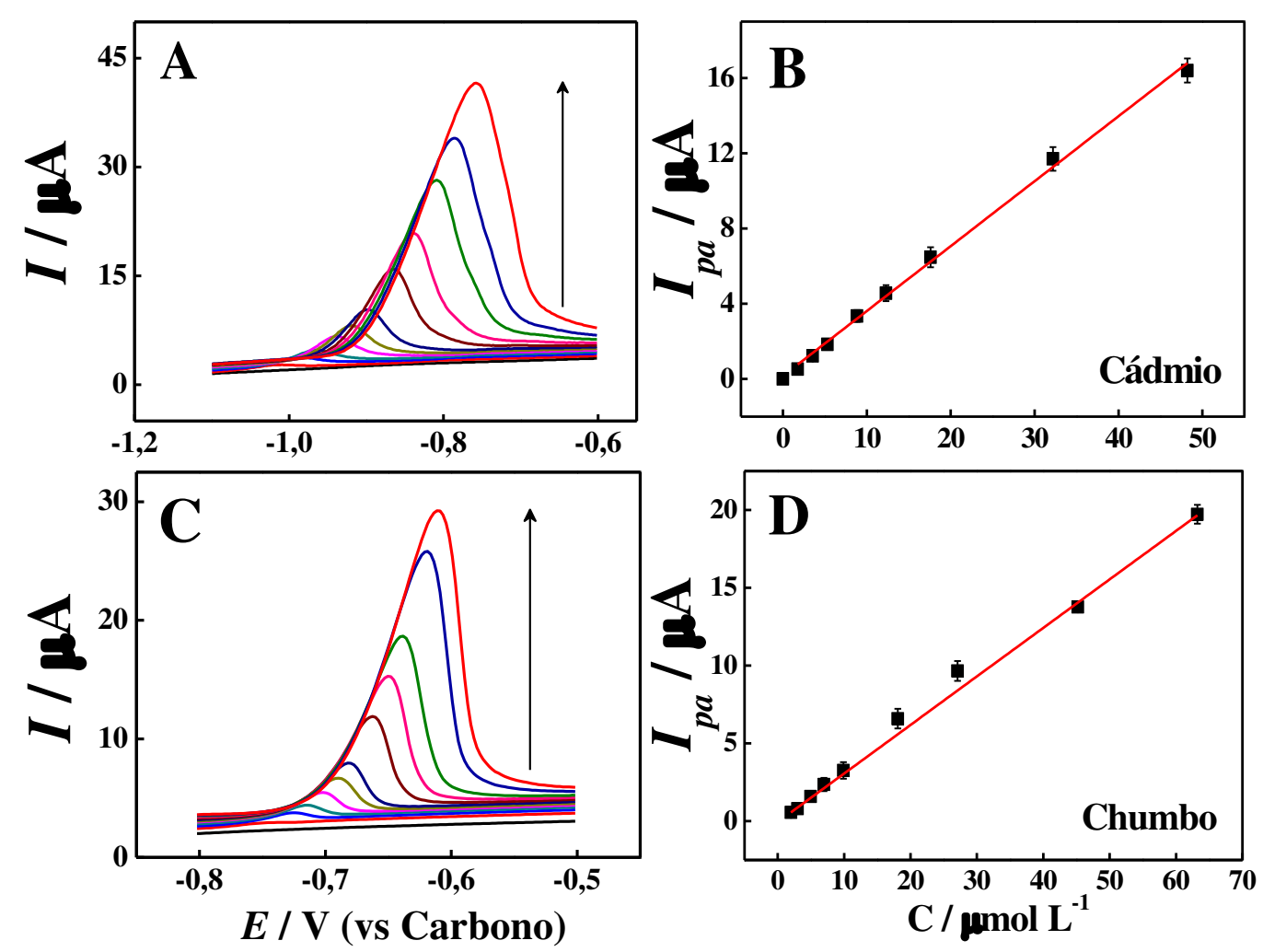

Figura 6. Voltamogramas de varredura linear obtidos para o El com a composição de $75 \%$ de NF e $25 \%$ de acetato de celulose $(\mathrm{m} / \mathrm{m})$ como tinta condutora para o $\mathrm{Cd}$ (II) (A) e $\mathrm{Pb}$ (II) (C) para diferentes concentrações dos respectivos íons, de $4,0 \times 10^{-7} \mathrm{~mol} \mathrm{~L}^{-1}$ à $9,0 \times 10^{-5} \mathrm{~mol} \mathrm{~L}^{-1}$ para o $\mathrm{Cd}(\mathrm{II})$ e de $8,0 \times 10^{-7} \mathrm{~mol} \mathrm{~L}^{-1}$ a $1,6 \times 10^{-4} \mathrm{~mol} \mathrm{~L}^{-1}$ para o $\mathrm{Pb}(\mathrm{II})$. Curva de correlação entre a intensidade de corrente de pico anódico para o $\mathrm{Cd}$ (II) (B) e $\mathrm{Pb}$ (II) (D). Tampão acetato pH 5,0 potencial de redução de $-1,3 \vee$ durante 60 segundos para íons $\mathrm{Cd}(\mathrm{II})$

Com base nas curvas analíticas dos respectivos íons pode-se obter informações quanto a desempenho analítico do eletrodo proposto para a determinação de íons $\mathrm{Cd}(\mathrm{II}) \mathrm{e}$ $\mathrm{Pb}(\mathrm{II})$. A faixa de resposta linear foi verificada para valores de concentração de $8,0 \times 10^{-7}$ $\mathrm{mol} \mathrm{L}^{-1}$ à $3,2 \times 10^{-5} \mathrm{~mol} \mathrm{~L}^{-1}$ para o $\mathrm{Cd}(\mathrm{II})$ segundo a equação $I_{a} / \mu A=0,033+0,349 C$ $\left(\mu \mathrm{mol}^{-1}\right)$ e de $1,0 \times 10^{-6} \mathrm{~mol} \mathrm{~L}^{-1}$ à $6,3 \times 10^{-5} \mathrm{~mol}$ $\mathrm{L}^{-1}$ para o $\mathrm{Pb}(\mathrm{II})$ segundo a equação $\mathrm{I}_{\mathrm{a}} / \mu \mathrm{A}=$ $0,056+0,312 C\left(\mu \mathrm{mol}^{-1}\right)$; sensibilidade de 0,349 e $0,312 \mu \mathrm{A} \mathrm{L} \mathrm{mol}^{-1}$ para o íons Cd(II) e $\mathrm{Pb}(\mathrm{II})$, respectivamente; limites de detecção ${ }^{21}$ de $5,8 \times 10^{-7} \mathrm{~mol} \mathrm{~L}^{-1}$ para o $\mathrm{Cd}(\mathrm{II})$ e $6,8 \times 10^{-7}$ mol $\mathrm{L}^{-1}$ para $\mathrm{O} \mathrm{Pb}(\mathrm{II})$; e limites de quantificação ${ }^{21}$ de $1,9 \times 10^{-6} \mathrm{~mol} \mathrm{~L}^{-1}$ para o $\mathrm{Cd}(\mathrm{II})$ e $2,3 \times 10^{-6} \mathrm{~mol} \mathrm{~L}^{-1}$ para o $\mathrm{Pb}(\mathrm{II})$.

Para melhor avaliar a potencialidade destes dispositivos, foi realizada a determinação simultânea de cádmio e chumbo em amostras de água de torneira enriquecidas com concentrações de íons cádmio (II) e chumbo (II) próximas aos limites estipulados pela resolução CONAMA $430 / 2011$ para estas espécies $\left(1,8 \times 10^{-6} \mathrm{~mol}\right.$ $\mathrm{L}^{-1}$ de $\mathrm{Cd}(\mathrm{II})$ e $2,4 \times 10^{-6} \mathrm{~mol} \mathrm{~L}^{-1}$ de $\left.\mathrm{Pb}(\mathrm{II})\right)$. As determinações foram realizadas em triplicata pelo método da adição de padrão usando a voltametria linear de redissolução anódica nas condições empregadas no estudo anterior (Figura 7). Com base nos voltamogramas obtidos e das respectivas intensidades de corrente de pico anódico foi possível realizar a determinação de cada espécie. Os valores médios encontrados para as amostras de água de torneira enriquecidas apresentaram boa recuperação sendo estatisticamente concordantes a um nível de confiança de $95 \%$ (teste-t) para ambos os analitos. Os resultados evidenciaram a 
potencialidade e aplicabilidade dos Els para a determinação voltamétrica de íons $\mathrm{Cd}(\mathrm{II})$ e
$\mathrm{Pb}(\mathrm{II})$, simultaneamente, em amostras de água de torneira enriquecidas.
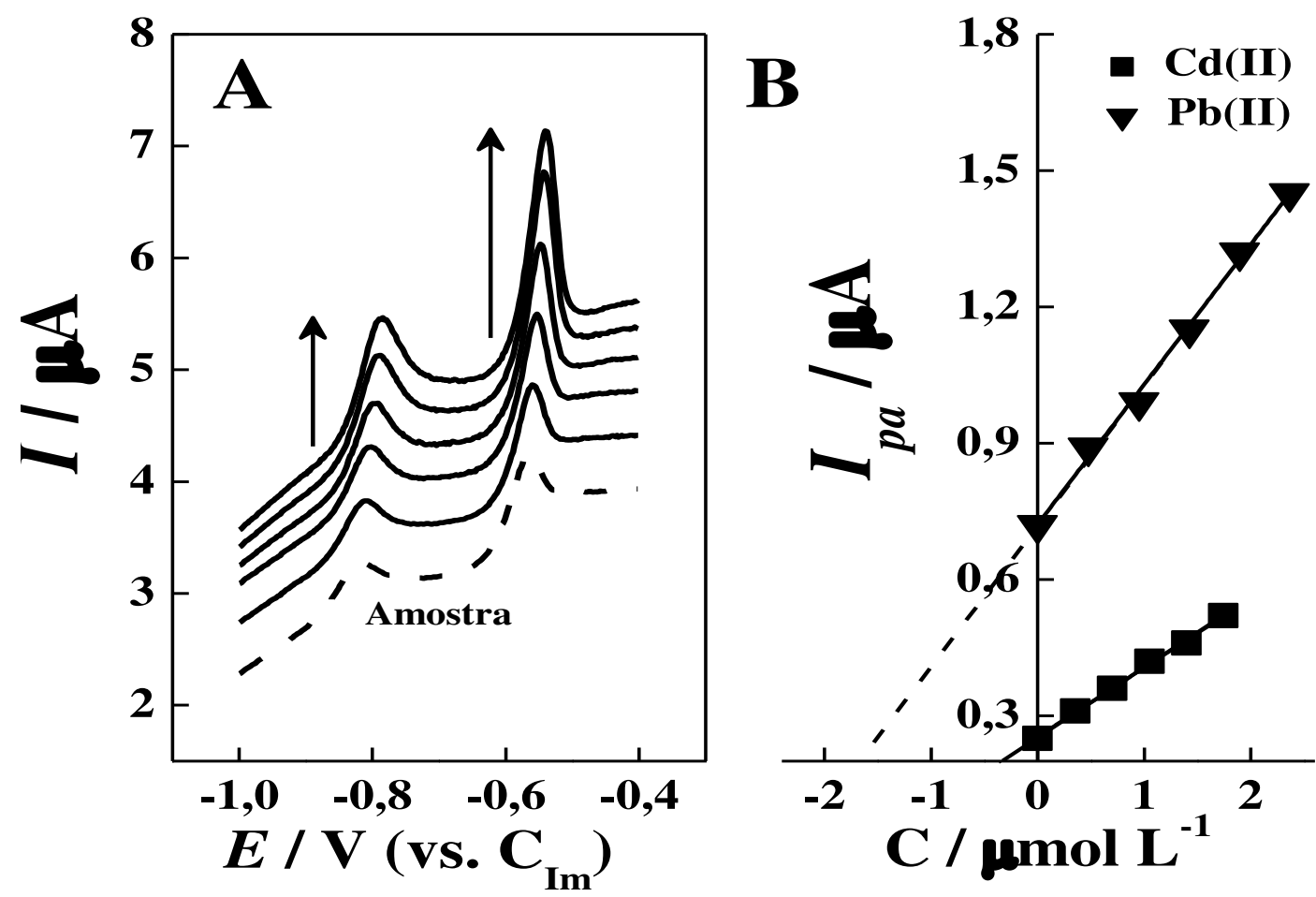

Figura 7. Voltamogramas de varredura linear obtidos para a amostra de água e após sucessivas adições dos padrões dos analitos (A) e as Curvas de correlação entre a intensidade de corrente e diferentes concentrações de íons $\mathrm{Cd}(\mathrm{II})$ e $\mathrm{Pb}$ (II) para a amostra enriquecida com $1,8 \times 10^{-6} \mathrm{~mol} \mathrm{~L}^{-1}$ de $\mathrm{Cd}(\mathrm{II})$ e $2,4 \times 10^{-6} \mathrm{~mol} \mathrm{~L}^{-1} \mathrm{de} \mathrm{Pb}(\mathrm{II})$ (B)

3.3. Determinação cronoamperométrica de ácido ascórbico em formulações farmacêuticas

Para a avaliação da potencialidade analítica do El na determinação de espécies orgânicas foram obtidos voltamogramas cíclicos na ausência e na presença de 5,0 × 10${ }^{4} \mathrm{~mol} \mathrm{~L}^{-1}$ de ácido ascórbico (AA), Fig 8-A. Nesse voltamograma não são verificados sinais faradaicos na ausência do ácido ascórbico, demonstrando que o eletrodo não é eletroativo nesse intervalo de potencial. Em solução contendo ácido ascórbico é verificado um sinal em $0,38 \mathrm{~V}$ com comportamento irreversível típico do ácido ascórbico. Para este estudo foi utilizado como eletrólito suporte acetato de sódio 0,1 $\mathrm{mol} \mathrm{L}^{-1}$ com pH ajustado em 3,0 e velocidade de varredura de $30 \mathrm{mV} \mathrm{s}^{-1}$. Posteriormente, 0 desempenho do eletrodo na determinação de AA foi explorado empregando cronoamperometria (Fig 8-B). Nesse sistema, um potencial fixo é aplicado ao eletrodo de trabalho em uma célula sob agitação controlada, e sucessivas adições de AA fornecem um aumento da corrente anódica proporcional a concentração do analito. Uma curva analítica (Fig $8-C$ ) para diferentes concentrações de AA $\left(1,0 \times 10^{-6}\right.$ a $5,7 \times 10^{-4}$ mol $\mathrm{L}^{-1}$ ) foi construída com as condições otimizadas, eletrólito suporte $0,1 \mathrm{~mol} \mathrm{~L}^{-1}$ de ácido acético ajustado em $\mathrm{pH} 3,0$, e potencial aplicado de $+0,55 \mathrm{~V}$ (vs. $\mathrm{C}_{\text {Impresso }}$ ). 


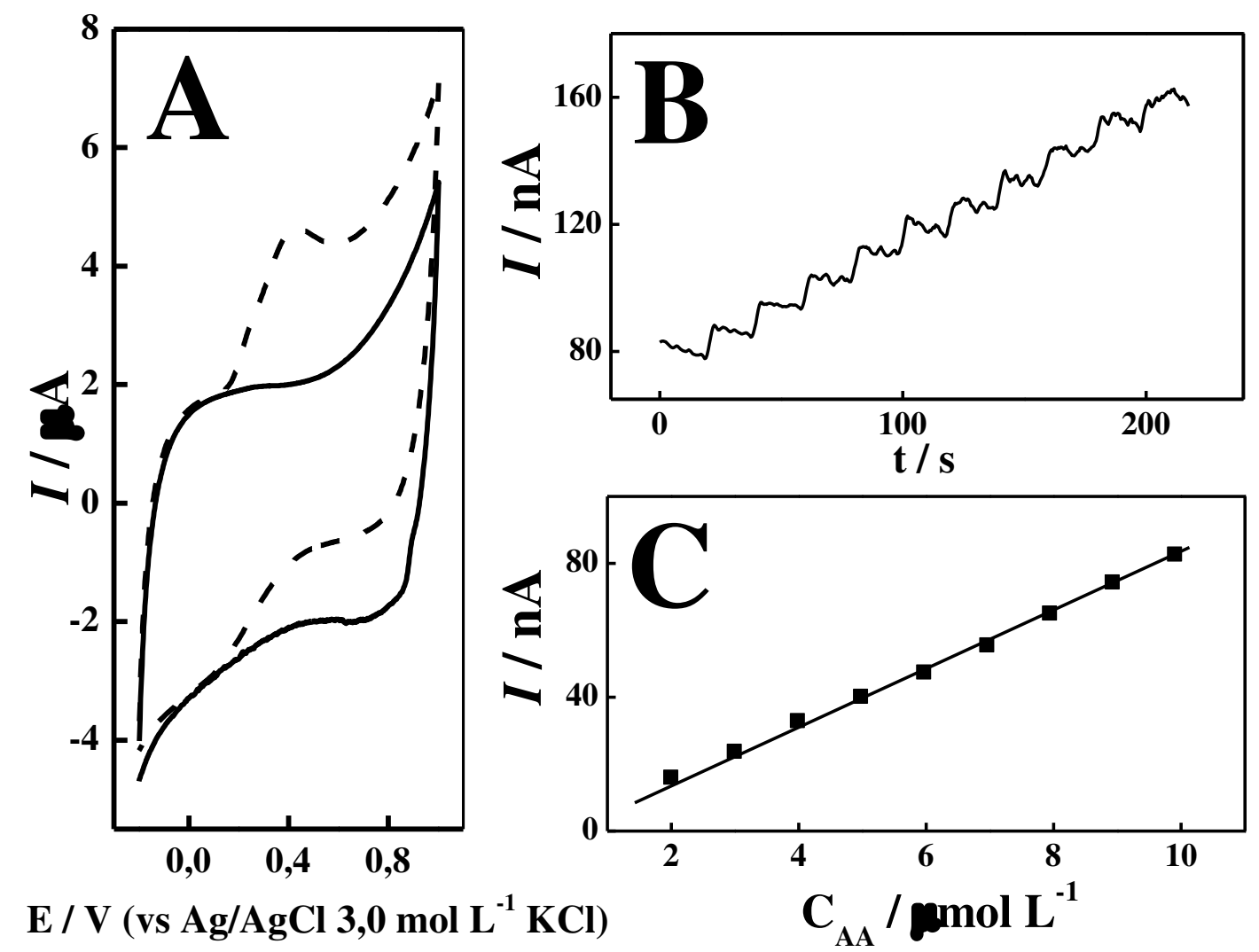

Figura 8. Voltamogramas cíclicos obtidos para o El na ausência (-) e na presença (---) de 5,0 $x$ $10^{-4} \mathrm{~mol} \mathrm{~L}^{-1}$ de $A A$, velocidade de varredura de $30 \mathrm{mV} \mathrm{s}^{-1}(\mathrm{~A})$; Curva cronoamperométrica obtida com o El após sucessivas adições de $A A\left(1,0 \times 10^{-6}\right.$ a $\left.5,7 \times 10^{-4} \mathrm{~mol} \mathrm{~L}^{-1}\right)$ aplicando o potencial de $+0,55 \mathrm{~V}(\mathrm{~B})$; Curva de correlação ( vs $\mathrm{C}_{\mathrm{AA}}$ ) referente a curva analítica (C)

Com base nos resultados obtidos foi observada boa linearidade $\left(R^{2}=0,998\right.$ (coeficiente de determinação) entre a corrente e a concentração de AA para o intervalo de concentrações entre $2,0 \times 10^{-6} \mathrm{e}$ $1,0 \times 10^{-5} \mathrm{~mol} \mathrm{~L}^{-1}$ com a equação da reta I / nA $=0,76+84,2[\mathrm{AA}] /$ ] $\mathrm{mol} \mathrm{L}^{-1}$. Obteve-se uma sensibilidade de $8,4 \mathrm{nA} \mathrm{L} \mathrm{mmol}^{-1}$, limite de detecção ${ }^{21}$ de $5,7 \times 10^{-7} \mathrm{~mol} \mathrm{~L}^{-1}$ e limite de quantificação ${ }^{21}$ de $1,9 \times 10^{-6} \mathrm{~mol} \mathrm{~L}^{-1}$.

Após verificada a potencialidade analítica do El na determinação amperométrica de AA, o procedimento proposto foi aplicado na determinação de AA em formulações farmacêuticas de vitamina $C$ efervescente. A quantificação de três amostras foi realizada em triplicata por adição de padrão, e os valores de concentração para cada amostra determinadas pelo método proposto foram comparados com os valores encontrados pelo método oficial estipulado pela Farmacopéia americana ${ }^{22}$ (titulação iodimétrica). A comparação entre os valores obtidos para os dois métodos citados e os valores rotulados para cada amostra está exemplificada na Tabela 3.

O método, além de se mostrar simples, rápido (cerca de 200 s) e eficiente, apresentou-se também preciso na determinação de AA em formulações farmacêuticas, uma vez apresentou valores concordantes quando comparados aos valores rotulados e obtidos pelo método comparativo de acordo com o teste-t aplicado a um nível de confiança de $95 \%$. 
Tabela 3. Determinação de ácido ascórbico em produtos farmacêuticos empregando-se o sistema proposto e o método por iodimétria (método de referência) e seus respectivos valores de desvio padrão $(n=3)$

\begin{tabular}{cccccc}
\hline Amostras & Rotulado* & Referência & $\mathrm{Er}_{1} / \%$ & Proposto & $\mathrm{Er}_{2} / \%$ \\
\hline A & 1,00 & $0,96 \pm 0,03$ & $-1,08$ & $0,95 \pm 0,09$ & $-4,59$ \\
B & 1,00 & $0,93 \pm 0,08$ & $+1,90$ & $0,95 \pm 0,08$ & $-4,56$ \\
C & 1,00 & $0,94 \pm 0,07$ & $-1,09$ & $0,93 \pm 0,06$ & $-6,54$ \\
\hline
\end{tabular}

* valores em gcomprimido ${ }^{-1}$

Er1 $=\frac{\text { Proposto }- \text { Referência }}{\text { Referência }} \times 100$

Er2 $=\frac{\text { Proposto }- \text { Rotulado }}{\text { Rotulado }} \times 100$

\section{Conclusão}

O processo de confecção dos El's por serigrafia se mostrou simples e eficiente para a construção de eletrodos impressos com reprodutibilidade adequada para permitir a construção de um elevado número de dispositivos com um baixo custo de produção. O uso de uma tinta condutora baseada apenas no negro de fumo como componente condutor é um aspecto inovador para esse material, o que amplia sua potencialidade e torna o custo de produção ainda menor, sendo a composição de $75 \%$ de NF e $25 \%$ de acetato de celulose $(\mathrm{m} / \mathrm{m})$ mais adequada, no que se refere a viscosidade e homogeneidade, para o processo de impressão sobre o substrato de PVC.

Os El's desenvolvidos apresentaram potencialidade analítica para a determinação de íons $\mathrm{Cd}(\mathrm{II})$ e $\mathrm{Pb}$ (II) em concentrações que se encontraram dentro dos limites estipulados pela resolução do CONAMA 430/2011 para lançamento de efluentes. Além disso, também foi observada potencialidade do emprego destes dispositivos na determinação cronoamperométrica de $A A$, sendo encontrados valores de concentração concordantes com o método comparativo (iodimetria) e os valores rotulados na amostra.

\section{Agradecimentos}

Os autores agradecem as agências de fomento: Capes, CNPq e Fundação Araucária.

\section{Referências Bibliográficas}

${ }^{1}$ Li, M.; Li, Y.-T.; Li, D.-W. ; Long, Y.-T. Recent developments and applications of screenprinted electrodes in environmental assays-A review. Analytica Chimica Acta 2012, 734, 31. [CrossRef]

${ }^{2}$ Renedo, O. D.; Alonso-Lomillo, M. A.; Martínez, M. J. A. Recent developments in the field of screen-printed electrodes and their related applications. Talanta 2007, 73, 202. [CrossRef]

${ }^{3}$ Gai, M.; Frueh, J.; Sukhorukov, G. B.; GirardEgrot, A.; Rebaud, S.; Doumeche, B.; He, Q. Microcontact printing of polyelectrolyte multilayer thin films: Glass-viscous flow transition based effects and hydration methods. Colloids and Surfaces $A$ : Physicochemical and Engineering Aspects 2015, 483, 271. [CrossRef]

${ }^{4}$ Man, Y.; Chen, Z.; Zhang, Y. ; Guo, P. Patterned growth of vertically aligned carbon nanotube arrays using colloidal lithography and plasma enhanced chemical vapor deposition. Journal of Alloys and Compounds 2015, 650, 86. [CrossRef] 
${ }^{5}$ Pavinatto, F. J.; Paschoal, C. W. A. ; Arias, A. $C$. Printed and flexible biosensor for antioxidants using interdigitated ink-jetted electrodes and gravure-deposited active layer. Biosensors and Bioelectronics 2015, 67, 553. [CrossRef]

${ }^{6}$ Soltani, N.; Haddadi, H.; Asgari, M. ; Rajabzadeh, N. Adsorptive stripping voltammetric detection of thorium on the multi-walled carbon nanotube modified screen printed electrode. Sensors and Actuators B: Chemical 2015, 220, 1212. [CrossRef]

${ }^{7}$ Albareda-Sirvent, M.; Merkoçi, A. ; Alegret, $S$. Configurations used in the design of screen-printed enzymatic biosensors. A review. Sensors and Actuators B: Chemical 2000, 69, 153. [CrossRef]

${ }^{8}$ Hart, J. P. ; Wring, S. A. Recent developments in the design and application of screen-printed electrochemical sensors for biomedical, environmental and industrial analyses. TrAC Trends in Analytical Chemistry 1997, 16, 89. [CrossRef]

${ }^{9}$ Alonso-Lomillo, M. A.; Domínguez-Renedo, O.; Arcos-Martínez, M. J. Recent developments in the design and application of screen-printed electrochemical sensors for biomedical, environmental and industrial analyses. Talanta 2010, 82, 1629. [CrossRef]

${ }^{10}$ Zhang, H.; Gao, M. ; Yang, X. Electrochemical oxidation and detection of paeonol on modified electrode with acetylene black nanoparticles. Colloids and Surfaces B: Biointerfaces 2011, 87, 378. [CrossRef]

${ }^{11}$ Wring, S. A.; Hart, J. P. Chemically modified, screen-printed carbon electrodes. Analyst 1992, 117, 1281. [CrossRef]

${ }^{12}$ Nascimento, V. B. ; Angnes, L. Eletrodos fabricados por "silk-screen". Química Nova 1998, 21, 614. [CrossRef]

${ }^{13} \mathrm{Cie}, \mathrm{C}$; 13 - Further opportunities with ink jet printed textiles, in Ink Jet Textile Printing, CIE, C., Editor. 2015, Woodhead Publishing. p. 165. [CrossRef]

${ }^{14}$ Sánchez-González, J.; Macías-García, A.; Alexandre-Franco, M. F. ; Gómez-Serrano, V. Electrical conductivity of carbon blacks under compression. Carbon 2005, 43, 741.[CrossRef]

${ }^{15}$ Huang, J. Carbon Black filled conducting polymers and polymer blends. Advances in Polymer Technology 2002, 21, 299. [CrossRef]

${ }^{16}$ Leong, C.-K. ; Chung, D. D. L. Carbon black dispersions and carbon-silver combinations as thermal pastes that surpass commercial silver and ceramic pastes in providing high thermal contact conductance. Carbon 2004, 42, 2323. [CrossRef]

17 Heinrich, G.; Klüppel, M. ; Vilgis, T. A. Reinforcement of elastomers. Current Opinion in Solid State and Materials Science 2002, 6, 195. [CrossRef]

${ }^{18}$ Beebe, R. A.; Biscoe, J.; Smith, W. R. ; Wendell, C. B. Heats of Adsorption on Carbon Black. I. Journal of the American Chemical Society 1947, 69, 95. [CrossRef]

${ }^{19}$ Lin, L.; Yang, J.; Lin, R.; Yu, L.; Gao, H.; Yang, $\mathrm{S}$. ; Li, X. In vivo study on the monoamine neurotransmitters and their metabolites change in the striatum of Parkinsonian rats by liquid chromatography with an acetylene black nanoparticles modified electrode. Journal of Pharmaceutical and Biomedical Analysis 2013, 72, 74. [CrossRef]

${ }^{20}$ Zheng, D.; Liu, X.; Zhou, D. ; Hu, S. Sensing of nitric oxide using a glassy carbon electrode modified with an electrocatalytic film composed of dihexadecyl hydrogen phosphate, platinum nanoparticles, and acetylene black. Microchim Acta 2012, 176, 49. [CrossRef]

${ }^{21}$ Talarico, D.; Arduini, F.; Constantino, A.; Del Carlo, M.; Compagnone, D.; Moscone, D. Carbon black as successful screen-printed electrode modifier for phenolic compound detection. Electrochemistry Communications 2015, 60, 78. [CrossRef]

${ }^{22}$ Miller, J. N.; Miller, J. C.; Statistics and Chemometrics for Analytical Chemistry. 6a. ed., Pearson: England 2010.

${ }^{23}$ USP, United States Pharmacopeia The National Formulary. 27 ed. 32. 2009, 27, Rockville: United State Pharmacopeia Convention.[CrossRef] 